\title{
Correlative Analysis of Long Term Cosmic Ray Variation in Relation with Interplanetary Magnetic Field
}

\author{
Sarver A. Khan $^{1 *}$, A. K.Saxena ${ }^{2}$, C.M. Tiwari ${ }^{3}$ \\ ${ }^{1}$ Department of physics A.P.S University (M.P), India \\ ${ }^{2}$ Department of physics A.P.S University (M.P), India \\ ${ }^{3}$ Department of physics A.P.S University (M.P), India
}

Available online at: www.isroset.org

Received: 24/Mar/2019, Accepted: 28/Apr/2019, Online: 30/Apr/2019

\begin{abstract}
In this paper we have performed the relationship between cosmic ray intensity (CRI) and Interplanetary Magnetic field (B) for the period 1976 to 2018. For this we have taken the data of Cosmic Ray Intensity (CRI) from various Neutron Monitor Stations, which are well maintained stations and provide reliable Cosmic Ray Data. Here we have taken the monthly and annual mean value of Cosmic Ray Intensity (CRI) from the Moscow (2.42GV), Russia Neutron Monitor Station. It has been found that Interplanetary Magnetic field (B) shows anti-phase with Cosmic Ray Intensity (CRI). It is found that Interplanetary Magnetic Field (IMF-B) shows decreasing trend with CRI and also shows negative correlation. Also we have taken interplanetary magnetic field (B), sunspot number (SSN) and $A_{p}$ index from omni web.
\end{abstract}

Keywords- Cosmic Ray Intensity (CRI), Interplanetary Magnetic Field (IMF-B). Sunspot number (SSN), $A_{p}$ index

\section{INTODUCTION}

The energetic particles traditionally known as cosmic rays are believed to be produced by a number of different sources, such as the remnants of supernovae, neutron stars, and black holes and sometimes from the more exotic objects from the sun and stars and also from the abandoned objects of radio galaxies. They undergo collisions with atoms of the upper atmosphere and produce a cascade of secondary particles that shower down through the atmosphere onto the surface of the Earth. (See, e.g., Hess 1912; Kohlhorster 1913; Millikan and Bowen 1926; Rossi 1964; Hillas 1972; Gaisser 1990; Longair 1992). Cosmic rays are of galactic and extragalactic origin though small parts of cosmic rays (CR) are originated from sun as protons, alpha particles and heavier elements. Cosmic ray intensity (CRI) is almost constant outside the heliosphere but modulation happens during their passage through the heliosphere due to interplanetary magnetic field [Mavromichalaki et. al., 1988; Agarwal et. al., 1993]. Their information is obtained from ground based neutron monitors. $(\mathrm{RC}=2.42)$ Forbush decreases (FDs), are short-term decreases in the intensity of galactic cosmic rays that were first observed by Forbush (1937, 1938). Solar indices are the markers of solar activity along with Sunspot number and $A_{p}$ index. Similar to earth; the sun has a cycle of about 11 years. Forbush first pointed out the inverse link between Sunspot number and cosmic ray intensity [Forbush, 1958; Rao, 1972; Morral, 1976; King, 1979; Biber et. al., 1983; Singh et. al., 2013].The value of correlation varies from one cycle to another. Many research groups have tried to explain this long-term modulation of the Cosmic ray intensity through means of various solar indices such as sunspots numbers (SSN), interplanetary magnetic field (B) and $A_{p}$ index. The modulation of galactic cosmic rays in the heliosphere using theoretical as well as empirical approaches is successful and advanced rapidly [Potgieter, 1998]. The solar activity, being numerically expressed with relative sunspots numbers (SSN), interplanetary magnetic field (B) and $A_{p}$ index varies with time for a period of about eleven years. Although this period defines the solar activity cycle, it is shorter than 11 years for some cycles, but longer than 11 years for other cycles. Furthermore, the solar activity is usually higher for the cycles with the period shorter than 11 years as compared with the one with the period longer than 11 years. So, the degree of the solar activity is usually dependent on the length of the solar activity cycle [Sakurai, 1977; Sakurai et al 2009]. The 11 year variation in Cosmic ray Intensity observed on the earth is anti-correlated with sunspot number (SSN), interplanetary magnetic field (B) and $A_{p}$ index [Forbush, 1954]. The cosmic ray intensity varies inversely with sunspot numbers (SSN), interplanetary magnetic field (B) and $A_{p}$ index, showing maximum intensity at time when solar activity is the minimum in the 11- year sunspot cycle (Forbush 1954, 1958). However only one parameter is insufficient to explain cosmic ray modulation. It is an integrated factor which play significant role in cosmic ray modulation. 
In this paper we have made an attempt to correlate cosmic ray intensity (CRI) with sunspot number (SSN), interplanetary magnetic field (B) and $A_{p}$ index to explain the momentary behavior of cross correlation function with respect to solar activity parameters.

\section{DATA AND METHOD OF ANALYSIS}

Correlation between Cosmic ray intensity and Solar activity parameters are computed from pressure corrected data of Moscow Neutron monitor station (cut off rigidity $2.42 \mathrm{GV}$ ) and Sunspot Number(SSN), Interplanetary magnetic field (B), and $A_{p}$ index are taken by omniweb data centre.

Correlation coefficients are computed for the period 19762018. The error of each correlation coefficient $(\mathrm{R})$ is given by

$\mathrm{PE}=0.6745\left(1-R^{2}\right) / \sqrt{N}$

Where, $\mathrm{N}$ is the sample size.

Characteristic behaviors of Odd and Even Solar cycles are different for solar activity parameters. Correlation coefficient is high for Odd Solar cycles compared to Even Solar cycles is maximum between Cosmic ray intensity (CRI) and sunspot number (SSN), interplanetary magnetic field (B) and $A_{p}$ index.

The correlation coefficient between Cosmic ray intensity and different solar activity parameters has also been calculated for the Said period using the method of "minimizing correlation coefficient method".

In this paper we will find the correlation between Cosmic ray intensity (CRI) and interplanetary magnetic field (B), sunspot number (SSN) and $A_{p}$ index for the period 19762018.

Also we will see the relation between Cosmic ray intensity (CRI), and interplanetary magnetic field (B), sunspot number (SSN) and $A_{p}$ index. For present investigation, we have taken the data of interplanetary magnetic field (B), sunspot number $(\mathrm{SSN}), A_{p}$ index and Cosmic ray intensity for the period 1976-2018.

\section{RESULTS AND DICUSSION}

The sun and its outputs in the form of various interplanetary features such as sunspot number (SSN), interplanetary magnetic field (B), and $A_{p}$ index are related to the disturbances in earth magnetic field. Figure $(1,2)$ it shows the linear plot between monthly and yearly value of cosmic ray intensity (CRI) of Moscow Neutron monitor stations with Sunspot number for the period 1976-2018 respectively. From the analysis it is clear that cosmic ray intensity (CRI) and sunspot number are anti-phase. It is clear from Figure.3. that Cosmic ray intensity (CRI) shows anti- correlation with sunspot number (SSN) and correlation coefficient is found to be for Solar cycle $21=-0.63129$, Solar cycle $22=-$ 0.91416 , Solar cycle $23=-0.77333$ and Solar cycle $24=-$ 0.84079 for the period 1976-2018.

Similarly from Figure $(6,7$.$) and Figure.8. it is clear that$ Cosmic ray intensity (CRI) shows anti-phase with interplanetary magnetic field (B) and correlation coefficient between these two parameters is found to be for Solar cycle $22=-0.44999$, Solar cycle $23=-0.30671$ and Solar cycle 24 $=-0.6608$ where as Solar cycle 21, shows less correlation between Cosmic ray intensity (CRI) and interplanetary magnetic field (B) and correlation coefficient is $=0.291072$.

Figure $(9,10)$ shows linear plot between Cosmic ray intensity (CRI) with $A_{p}$ index. From Figures(11,12 \& 13) it is clear that Cosmic ray intensity also shows anti-correlation with $A_{p}$ index and correlation coefficient is found to be for Solar cycle $21=-0.4644$, Solar $22=-0.5630$, Solar cycle 23 $=-0.6223$ and Solar cycle $24=-0.3753$.

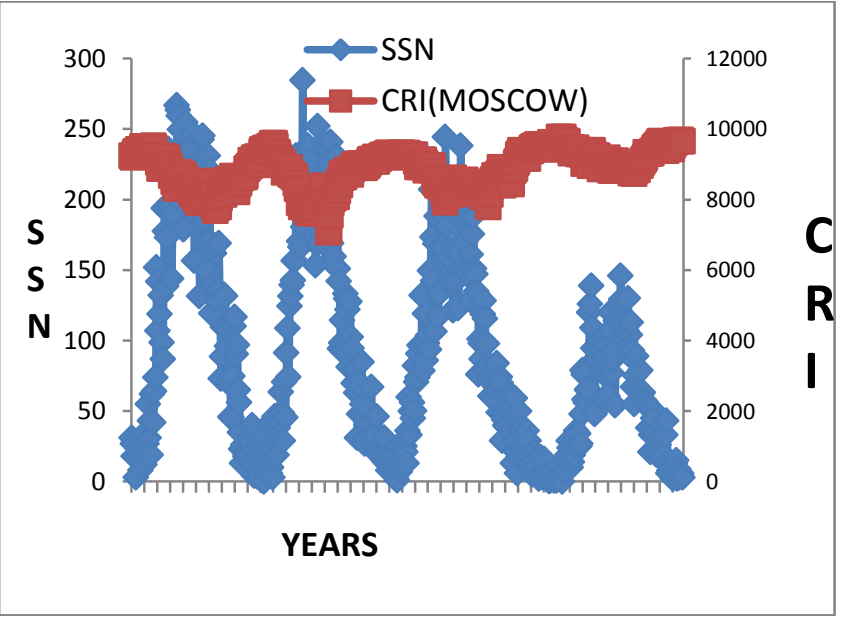

FIG.1.Shows monthly Linear plot between Cosmic ray intensity (CRI) of Moscow Neutron Monitor Station and Sunspot number (SSN) for the period 1976-2018.

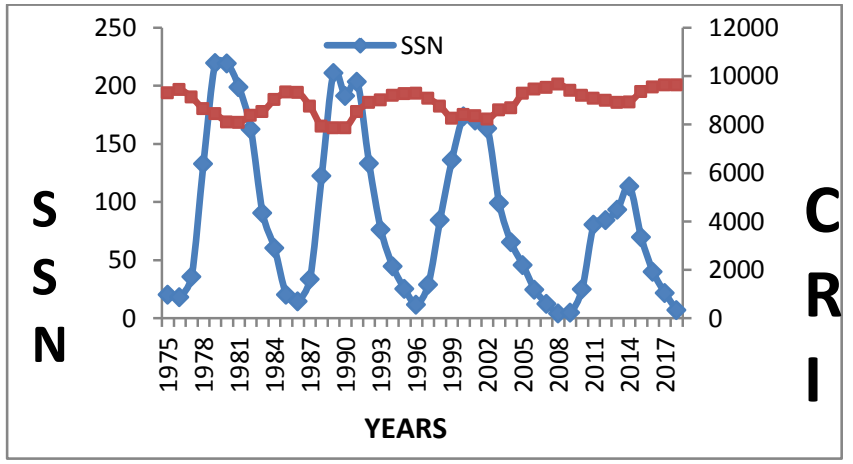

FIG.2.Shows yearly Linear plot between Cosmic ray intensity (CRI) of Moscow Neutron Monitor Station and Sunspot number (SSN) for the period 1976-2018. 


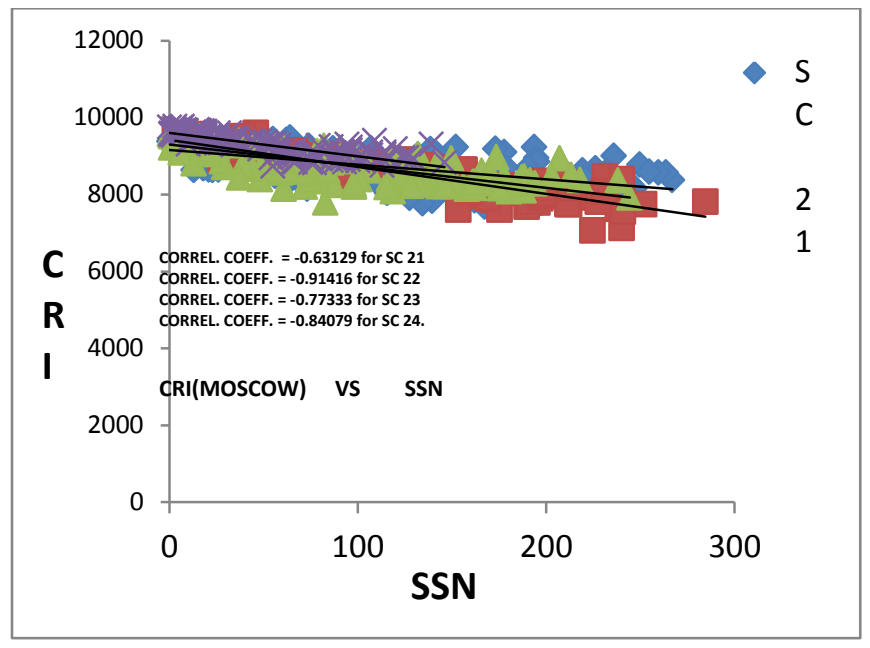

FIG.3. shows correlation between Cosmic ray intensity (CRI) of Moscow Neutron Monitor station and Sunspot number (SSN) for the period 19762018

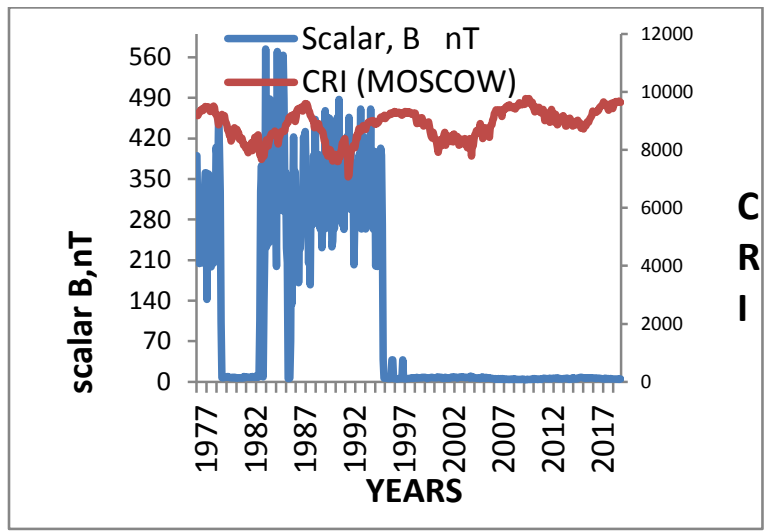

FIG.4. Shows monthly linear plot between Cosmic ray intensity (CRI) of Moscow Neutron Monitor Station and interplanetary magnetic field (B) for the period 1976-2018.

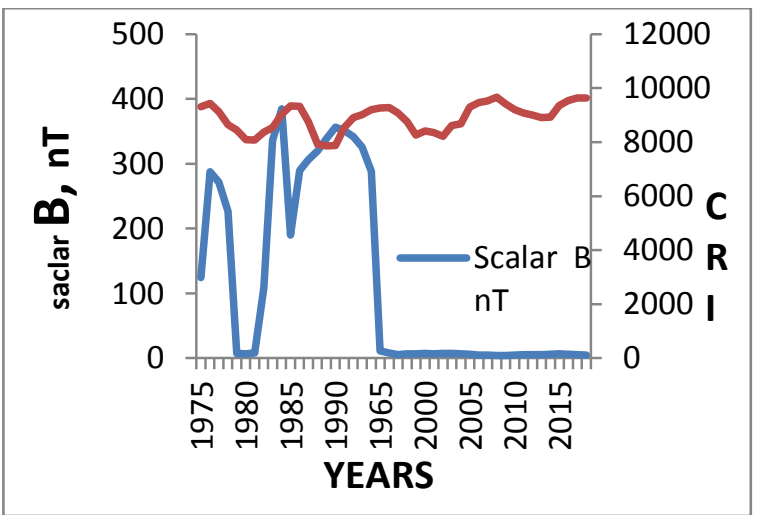

FIG.5. Shows yearly linear plot between Cosmic ray intensity (CRI) of Moscow Neutron Monitor Station and interplanetary magnetic field (B) for the period 1976-2018.

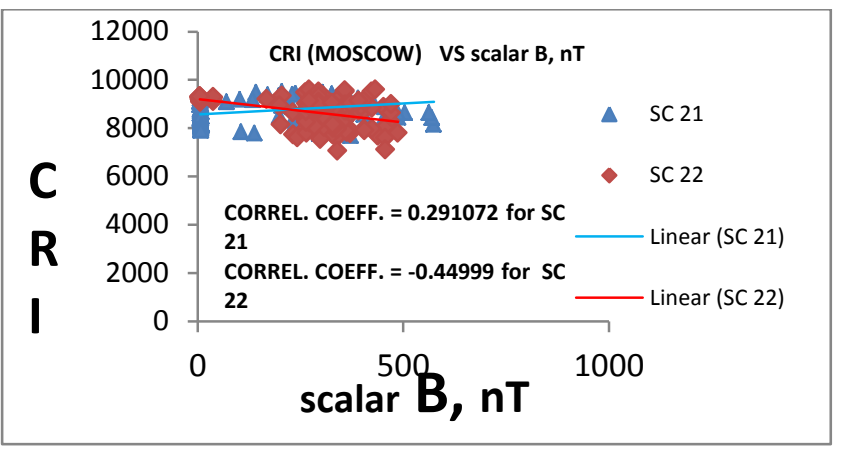

FIG.6. Show correlation between Cosmic ray intensity (CRI) of Moscow Neutron Monitor Station and IMP (B) for the period 1976-1996, i.e.; solar cycle 21 and solar cycle 22 .

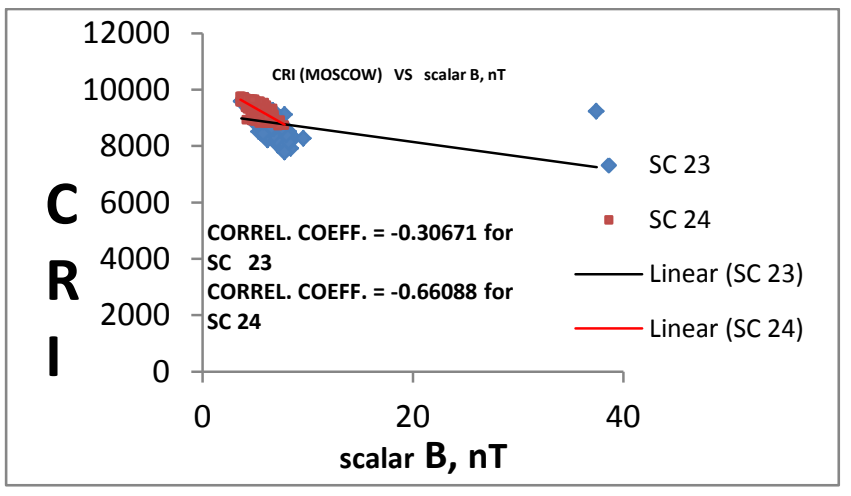

FIG.7. Show correlation between Cosmic ray intensity (CRI) of Moscow Neutron Monitor Station and IMP (B) for the period 1996-2018, i.e.; solar cycle 23 and solar cycle 24 .

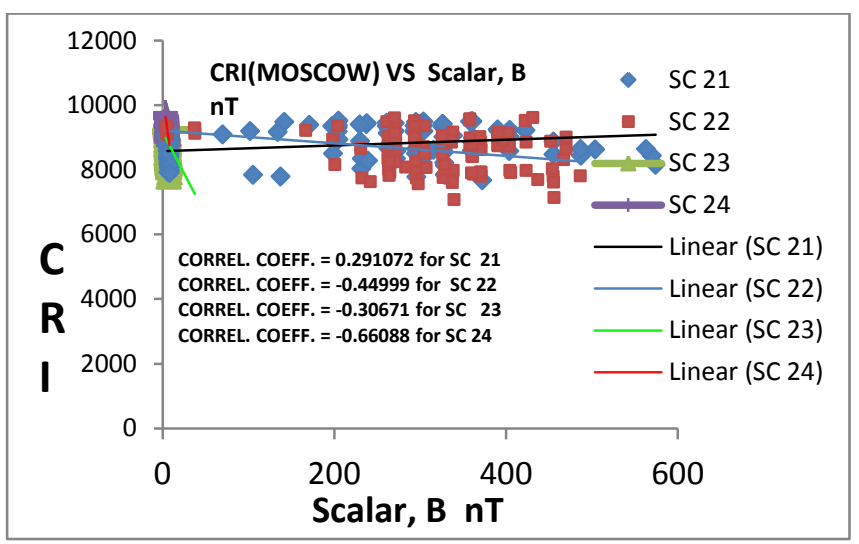

FIG.8. Show correlation between Cosmic ray intensity (CRI) of Moscow Neutron Monitor Station and IMP (B) for the period 1976-2018 


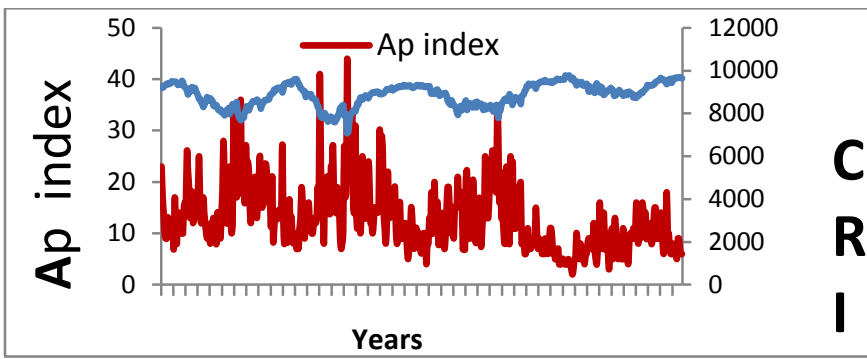

FIG.9. Shows monthly linear plot between Cosmic ray intensity (CRI) of Moscow Neutron Monitor Station and $A_{p}$ index for the period 1976-2018.

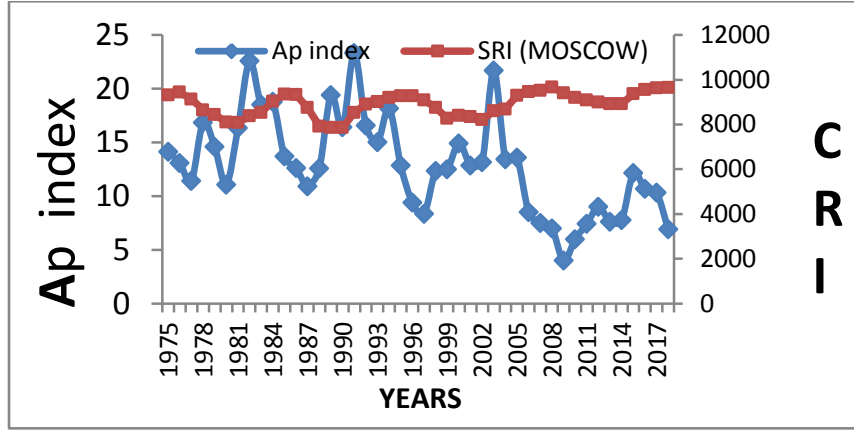

FIG.10. Shows yearly linear plot between Cosmic ray intensity (CRI) of Moscow Neutron Monitor Station and $A_{p}$ index for the period 1976-2018

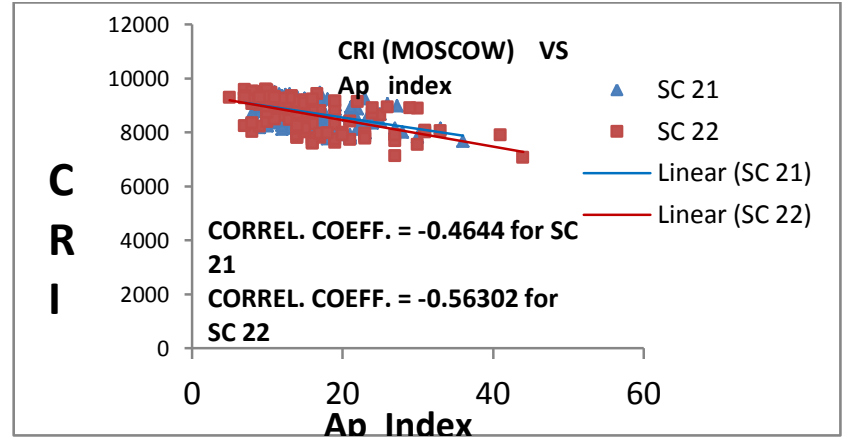

FIG.11. Show correlation between Cosmic ray intensity (CRI) of Moscow Neutron Monitor Station and $A_{p}$ index for the period 1976-1996, i.e.; solar cycle 21 and solar cycle 22 .

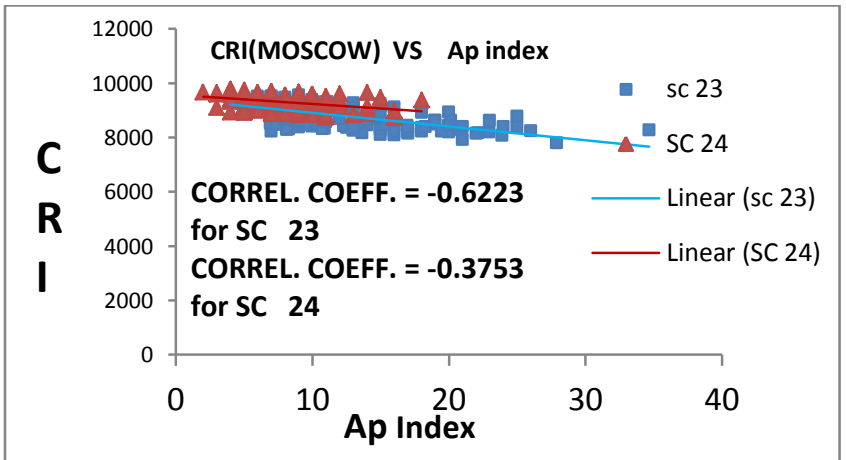

FIG.12. Show correlation between Cosmic ray intensity (CRI) of Moscow Neutron Monitor Station and $A_{p}$ index for the period 1996-2018, i.e.; solar cycle 23 and solar cycle 24 .

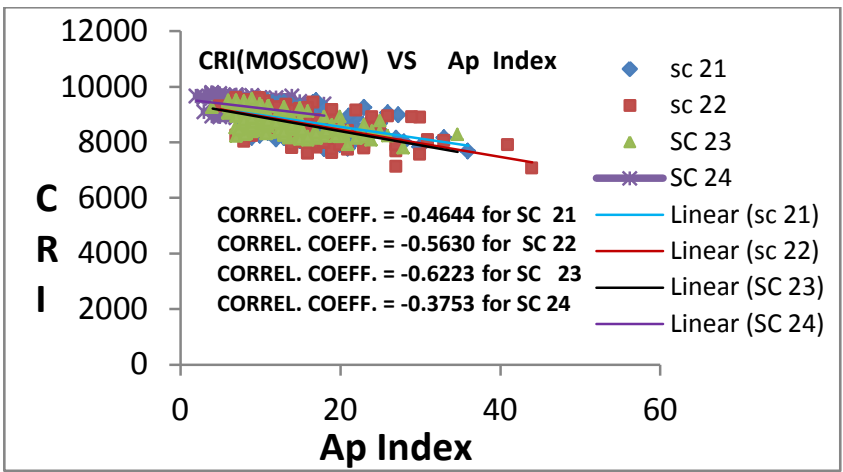

FIG.13. Show correlation between Cosmic ray intensity (CRI) of Moscow Neutron Monitor Station and $A_{p}$ index for the period 1976-2018

\section{CONCLUSION}

The variation of cosmic ray intensity during odd and even solar cycles give us information about the mechanism behind the ascending and descending phase of solar cycles 21, 22, 23 and 24 .Transport of cosmic ray by interplanetary magnetic field also have significant role .The anti correlation between cosmic ray intensity and solar activity parameter (Sunspot number) is smaller in odd solar cycles as compared to even solar cycles. The cosmic ray intensity and interplanetary magnetic field (B) be have less positive correlation in solar cycle 21 but anti phase with solar cycle 2223 and 24.The cosmic ray intensity anti phase with $A_{p}$ index.

Anomalous phenomenon in the solar modulation of cosmic ray in addition to the variation of anti phase for the even and odd solar cycles have been reported. Such phenomenons in the cosmic ray intensity have also been observed after solar maximum as well as in the declining in phases of earlier solar cycles. In General the study of solar cycle is difficult, so the study of this particularly violent solar cycle makes the whole study far more difficult. If the empirical modulation can give us a good standard deviation between the observation and calculation values, it would be very satisfactory results and this study could be used in future studies of subsequent solar cycles. This will be helpful in studying space weather forecasting. It is of theoretical interest to understand why some cycles are very active in the declining phase, and the high level of activity in the declining phase has practical implications for planting solar observations and forecasting space weather.

\section{REFERENCE}

[1]. Hess, V.F.: Phys. Z. 13, 1084(1912).

[2]. Hillas, Y.A.M.: Cosmic Rays. Pergamon Press, Oxford/New York (1972)

[3]. Gaisser, T.K.: Cosmic Rays and Particle Physics. Cambridge University Press, Cambridge (1990)

[4]. Kohlhorster, W.: Phys. Z. 14, 1153(1913) 
[5]. Longair, M.S.: Particles, Photons and their detection. In: High Energy Astrophysics, $2^{\text {nd }}$ edn. Vol.1. Cambridge University Press, Cambridge (1992)

[6]. Millikan, R.A., Bowen, I.S.: The Physical Review, Published as second series 27, 4(1926)

[7]. Rossi, B.: Cosmic Rays, Chap.1, P.1. McGraw-Hill, New York (1964)

[8]. Agrawal, S.P. Shrivastava, P.K., Shukla, R.P.(1993): Time depending solar cycle, Cosmic ray modulation of $23^{\text {rd }}$ neutron monitor energies. Proc, 23 Int. Cosmic Ray Conf., 3, 590.

[9]. Mavromichalaki, H., Marmatsouri, E., Vassilaki, A. (1988): Solar cycle phenomena in Cosmic ray intensity; Differences between even and odd cycles. Earth, Moon Planets, 42, 233244.

[10]. Forbush, S.E. 1937, Phys. Rev. 51, 1108

[11]. Forbush, S. E. 1938, Phys. Rev., 54, 975

[12]. Forbush, S. E. (1958): Cosmic ray intensity variations during two solar cycles. J. Geophys Res., 63, 651.

[13]. Moraal, H. (1976): Observation of the eleven year cosmic ray Modulation cycle. Space Sci. Rev., 19, 845-920.

[14]. Rao, U. R. (1972): Solar modulation of galactic cosmic radiation. Space Sci. Rev., 12, 719-809.

[15]. Biber, Pomerantz, Taso. (1983): C. M. Proc. 18th Int. Cos. Ray Conf., (Bangalore), 8, 289

[16]. King, J. H. (1979): Solar cycle variations in IMF intensity. J. Geophys. Res., 84, 5938-5940.

[17]. Singh, S. G., Saxena, A. K., Singh, R. P., and Singh, Y. K. (2013): Correlative study of solar wind streams velocity and cosmic ray intensity variations during 2002-2007. Int. J. Sci. Env.Tech., 2, 56.

[18]. M.S. Potgieter, "The modulation of galactic cosmic rays in the heliosphere: theory and Models," Space Science Reviews, vol. 83, no. 1-2, pp. 147-158, 1998.

[19]. K. Sakurai: Nature 296 (1977) 401.

[20]. Kunitomo Sakurai, Makoto Hareyama, Satoshi Kodaira and Nobuyuki Hasebe J. Phys. Soc. Jpn. Full J. Phys. Soc. Jpn. 78 (2009), Supplement A K. Sakurai et al. 11.

[21]. Forbush, S.E. 1954, J. Geophys. Res., 59,525

[22]. Forbush, S.E., 1958, J. Geophys. Res., 63, 651

[23]. S.singh, A.P. Mishra (2015) "interaction of solar plasma near earth with reference to geomagnetic storms during maxima of solar cycle 24" doi. 1007/S12648-015-0703-Y. 